\title{
ANTIBODY REPERTOIRE OF ATLANTIC SALMON: IG-SEC OR HIGH-THROUGHPUT SEQUENCING OF THE VARIABLE REGION OF IGM HEAVY CHAIN
}

\author{
Aleksei Krasnov ${ }^{1 S}$, Sergey Afanasyev ${ }^{1,2}$, Maria Dahle ${ }^{3}$, Sven Martin Jørgensen ${ }^{l}$, Morten Lund ${ }^{4}$, Espen \\ Rimstad $^{4}$ \\ ${ }^{l}$ Nofima AS, Norwegian Institutes of Food, Fisheries \& Aquaculture Research, Å, Norway \\ ${ }^{2}$ Sechenov Institute of Evolutionary Physiology and Biochemistry, Saint Petersburg, Russia \\ ${ }^{3}$ Section of Immunology, Norwegian Veterinary Institute, Oslo, Norway \\ ${ }^{4}$ Department of Food Safety and Infection Biology, Norwegian University of Life Sciences, Oslo,
} Norway

Immunoglobulins (Ig) play a key role in protection of fish against bacterial and viral pathogens. Atlantic salmon has three types of $\operatorname{Ig}(\mathrm{M}, \mathrm{D}$ and $\mathrm{T})$, of which IgM is the main actor of the adaptive humoral immunity. Ig heavy chains are encoded with two loci and their variable regions (VR) are produced by somatic recombination of $\mathrm{J}, \mathrm{D}$ and $\mathrm{V}$ genes. While immunological methods detect specific antibodies, parallel sequencing of VR (Ig-seq) provides a comprehensive survey of the repertoire. We established a protocol for construction of IgM-VR libraries for Illumina sequencing that captures all transcripts and a bioinformatics pipeline Iguana (Ig Universe Analyses). The clonotypes (CLT) of transcripts are identified by combinations of J, D and V genes and sequences of J(D)V junctions or CDR3 - the highly variable region, which is responsible for antigen binding. We also developed metrics for characterization of the repertoire. The size of salmon IgM-V repertoire is relatively large - to this end, more than 1.4 million different CLT have been detected in 18.5 million transcripts. An intriguing finding was a large size of the public compartment, i.e. CLT shared by at least several individuals. While a vast majority of CLT was unique, all of the most frequent transcripts were public and comprised a substantial fraction of IgM; i.e. 5.7\% CLT, which were found in at least two fish encoded $46.2 \%$ of all transcripts. The origin, properties and functional roles of public CLT in Atlantic salmon are unknown. It is possible that they are similar to natural polyreactive antibodies of warm blood animals, which are regarded as a part of the first line of defence against pathogens. Ig-seq was applied to monitor responses to viral infections (PMCV, PRV and SAV) and changes in course of Atlantic salmon development. Enhanced antibody production or clone expansion assessed by increased cumulative frequencies of most abundant CLT and Gini index was noticed after viral infection in the head kidney and to lesser extent in peripheral blood, but not in the spleen. Until present sequencing of IgM-V has not produced evidence for hyper mutations and affinity maturation. Communality or fraction of public CLT among the leaders showed markedly different changes after infection with SAV and PRV being respectively low in the former and high in the latter case.

Intriguingly, salmon infected with PRV showed high resistance to secondary infection with SAV and serum of PRV infected fish was able to neutralize SAV in vitro. Results suggest that public IgM transcripts may encode polyreactive antibodies that provide an unspecific protection.

Key words: Atlantic salmon, smoltification, breeding, immune suppression, transcriptome

${ }^{\S}$ Corresponding author. Telephone: +47 64970484. E-mail address: Aleksei.Krasnov@nofima.no 